ESAIM: M2AN

M2AN, Vol. 41, No 1, 2007, pp. 1-20

DOI: $10.1051 / \mathrm{m} 2 \mathrm{an}: 2007005$
ESAIM: Mathematical Modelling and Numerical Analysis

www.edpsciences.org/m2an

\title{
ON THE INF-SUP CONDITION FOR HIGHER ORDER MIXED FEM ON MESHES WITH HANGING NODES*
}

\author{
VinCENT Heuveline ${ }^{1,2}$ AND FRIEDHELM SCHIEWECK ${ }^{3}$
}

\begin{abstract}
We consider higher order mixed finite element methods for the incompressible Stokes or Navier-Stokes equations with $Q_{r}$-elements for the velocity and discontinuous $P_{r-1}$-elements for the pressure where the order $r$ can vary from element to element between 2 and a fixed bound $r^{*}$. We prove the inf-sup condition uniformly with respect to the meshwidth $h$ on general quadrilateral and hexahedral meshes with hanging nodes.
\end{abstract}

Mathematics Subject Classification. 65N30, 65N35.

Received: October 3, 2005. Revised: August 30, 2006.

\section{INTRODUCTION}

The use of higher order mixed finite elements is well established for the numerical approximation of incompressible flow problems. In the last decade, many efforts have been made in that context in order to combine judiciously the $h$-version of the finite element method (FEM) with the $p$-version or spectral type methods. For properly designed meshes, the resulting $h p$-FEM has been shown to give exponential rates of convergence even in the presence of singularities (see e.g. $[2,21]$ ). Typically the solution of incompressible Stokes or Navier-Stokes problems exhibits such singularities in the neighborhood of re-entrant corners (see e.g. [25] and references therein).

Due to the incompressibility constraint $\nabla \cdot u=0$, it is well known that the pair of finite element spaces used for the approximation of velocity and pressure can not be chosen arbitrarily. A compatibility condition known as the inf-sup condition has to be satisfied in order to guarantee stability and uniqueness of the discrete solution (see e.g. [9]). Under various assumptions on the underlying meshes, the inf-sup condition has been proven for many pairs of finite element spaces both for the $h$-version (see e.g. $[5,8,9,13,23]$ ) and the $p$-version (see e.g. $[3,4,10,24]$ ). However, this issue is more intricate for the case of $h p$-FEM which allows both local refinement by means of hanging nodes as well as various polynomial orders on the mesh elements. In the

\footnotetext{
Keywords and phrases. Inf-sup condition, higher order mixed finite element, adaptive grids, hanging nodes.

* The work of the first author was supported in part by the SFB 359 "Reaktive Strömungen, Diffusion und Transport" and in part by the BMBF (Bundesministerium für Bildung und For schung) project under grant 03.RAM.3HD.

1 Institute for Applied Mathematics II, University Karlsruhe (TH), Postfach 6980, 76128 Karlsruhe, Germany. vincent.heuveline@math.uni-karlsruhe.de

2 Scientific Supercomputing Center, University of Karlsruhe (TH), Zirkel 2, 76128 Karlsruhe, Germany.

vincent.heuveline@rz. uni-karlsruhe.de

3 Institut für Analysis und Numerik, Otto-von-Guericke-Universität Magdeburg, Postfach 4120, 39016 Magdeburg, Germany.

friedhelm.schieweck@mathematik.uni-magdeburg.de

(c) EDP Sciences, SMAI 2007
} 
two dimensional case, Schötzau et al. [20] have proven the inf-sup condition for the family of finite element pairs $\left(\mathbb{Q}_{r}, \mathbb{Q}_{r-2}\right)$ on so-called anisotropically and geometrically refined meshes which describe a prototypical local refinement hierarchy with hanging nodes. The cells of the mesh are assumed to be affine equivalent to a reference unit square or a reference unit triangle. Therefore, this analysis covers only the case where the quadrilateral elements are parallelograms. Ainsworth and Coggins [1] propose a uniformly stable family of finite element pairs $\left(\mathbb{Q}_{r}, \mathbb{Q}_{r-1}^{\prime}\right)$ where $\mathbb{Q}_{r-1}^{\prime}$ describes an augmented pressure space. While the velocity finite element functions associated with nodes at the element boundary are defined by means of the usual reference transformation, the functions corresponding to interior element nodes are based on the Piola transformation (see [10] for similar construction). In [1] an inf-sup condition for two dimensional meshes with hanging nodes has been proved where the inf-sup constant is uniformly bounded with respect to $h$ and the polynomial degree $r$.

In this paper, our goal is to prove the inf-sup condition for the family of finite element pairs $\left(\mathbb{Q}_{r}, \mathbb{P}_{r-1}^{\text {disc }}\right)$ on general quadrilateral and hexahedral meshes with hanging nodes where the polynomial degree $r$ may change from element to element between 2 and a maximum value $r^{*}$. By $\mathbb{P}_{r-1}^{\text {disc }}$ we denote the space of discontinuous functions which are elementwise defined by polynomials of degree less or equal to $r-1$. Our theory covers both the case of a "mapped" and "unmapped" pressure space (see [19] and [13], respectively) where "mapped" means that the finite element functions are defined by mapping polynomials from a reference element and "unmapped" means that the finite element functions are polynomials on the original element. On the one hand, concerning implementation aspects, the mapped pressure space is more attractive since the velocity space naturally is a mapped space too. On the other hand, the mapped pressure space may have non-optimal approximation properties on general quadrilateral or hexahedral meshes [5]. However, if the mesh is created from a regular coarse mesh only by means of recursive refinement of existing elements into $2^{d}$ many son-elements (where $d$ is the dimension of the domain) combined with the introduction of hanging nodes (see Sect. 2.2), then it has been shown that the mapped space has optimal approximation properties too [18].

For the finite element pair $\left(\mathbb{Q}_{r}, \mathbb{P}_{r-1}^{\text {disc }}\right)$ with the same polynomial degree $r$ on each element and a regular quadrilateral or hexahedral mesh without hanging nodes, it is already known (see [19] for the mapped and [13] for the unmapped pressure space) that the inf-sup condition holds uniformly with respect to the mesh size. In this paper, our main issue is the aspect of hanging nodes caused by non-matching mesh cells or non-matching polynomial degrees. We are interested in the question if hanging nodes affect the inf-sup condition in a negative way. The new contribution of this paper is the proof that on so-called 1-irregular meshes with hanging nodes the inf-sup condition holds uniformly with respect to the local and global mesh size and the number of hanging nodes.

Moreover, a main issue in our paper is that we consider general meshes consisting of non-affine equivalent elements defined by a multi-linear transformation of a reference element. Note that for such meshes in the three-dimensional case, the two-dimensional faces of the hexahedral elements can be curved in general. This, in particular, implies that the normal vector on a face is no longer constant which causes additional difficulties in the analysis. From the practical point of view, such general meshes with hanging nodes occur very naturally in applications with adaptive mesh refinement based on a posteriori error estimators (see e.g. $[15,16]$ ). Typical meshes are depicted in Figure 1.

It is well known (see [13]) that in both cases of modeling incompressible flows, by means of the Stokes equations as well as the Navier-Stokes equations, the divergence stability is guaranteed by the same condition. Therefore, in the sequel, we will consider as a model problem the Stokes equations with homogeneous Dirichlet conditions: Find a velocity field $u$ and a pressure distribution $p$ such that

$$
\begin{aligned}
-\nu \Delta u+\nabla p & =f & & \text { in } \Omega, \\
\operatorname{div} u & =0 & & \text { in } \Omega, \\
u & =0 & & \text { on } \Gamma .
\end{aligned}
$$

Here, $\Omega$ denotes a bounded domain in $\mathbb{R}^{d}, d \in\{2,3\}, \nu>0$ the kinematic viscosity and $f$ a given body force per unit mass. Then, a pair $\left(X^{N}, M^{N}\right)$ of conforming finite element spaces approximating the velocity $u \in\left(H_{0}^{1}(\Omega)\right)^{d}$ 

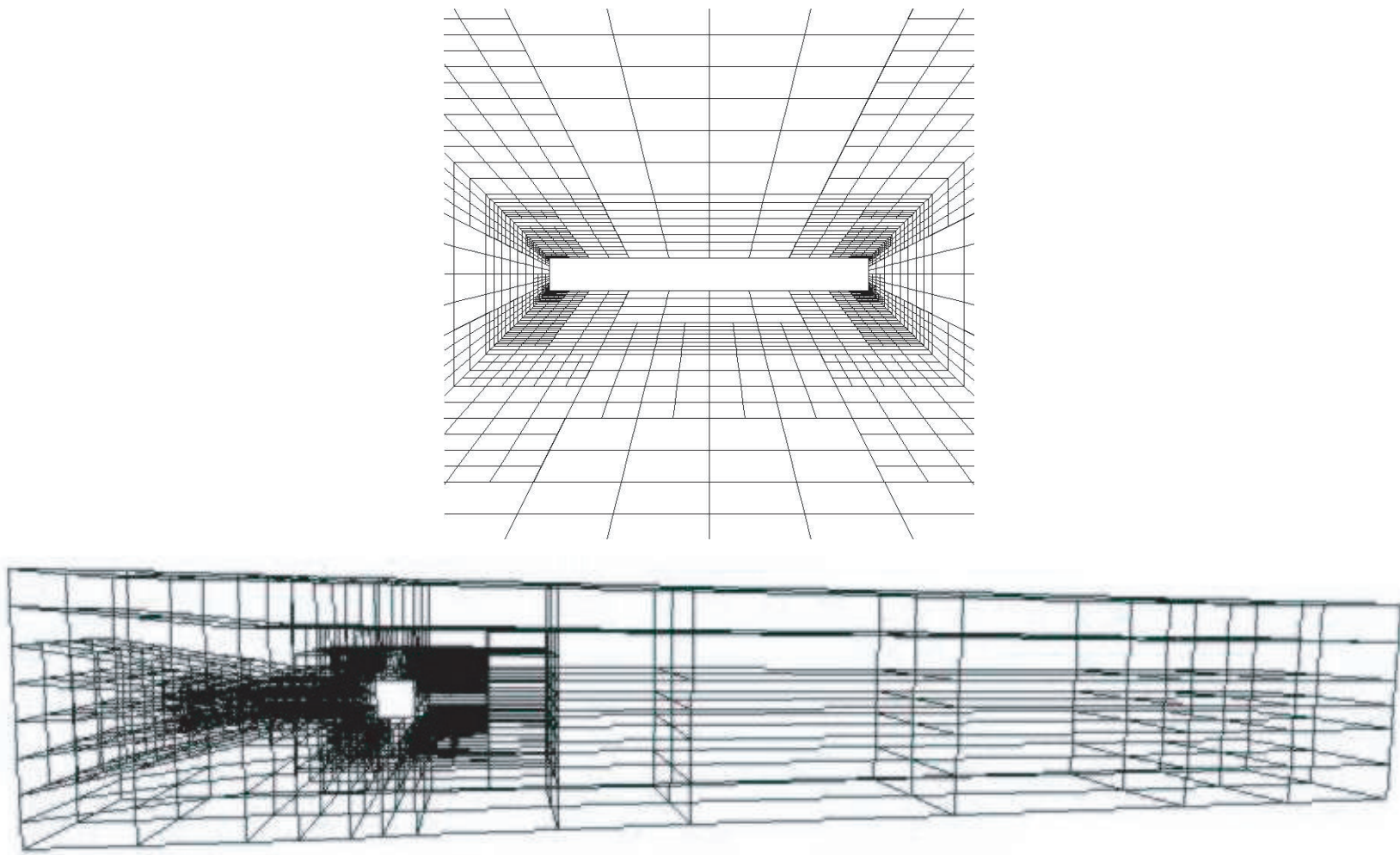

Hivision 2.0

Figure 1. Typical local refined meshes with hanging nodes: (top) two-dimensional grid for the computation of an exterior flow problem [7]; (bottom) three-dimensional grid for a channel flow problem [14].

and the pressure $p \in L_{0}^{2}(\Omega)$ is said to fulfill the discrete inf-sup condition if the following estimate holds:

$$
\exists \beta>0: \quad \inf _{0 \neq q^{N} \in M^{N}} \sup _{0 \neq v^{N} \in X^{N}} \frac{\left(\operatorname{div} v^{N}, q^{N}\right)}{\left|v^{N}\right|_{1, \Omega}\left\|q^{N}\right\|_{0, \Omega}} \geq \beta .
$$

It is desirable that the parameter $\beta$ in $(2)$ is independent of the mesh size and of the polynomial degree. For our family of finite element pairs $\left(\mathbb{Q}_{r}, \mathbb{P}_{r-1}^{\text {disc }}\right)$, we prove that the inf-sup condition holds with a constant $\beta\left(r^{*}\right)$ which is independent of the local and global mesh size but may depend on the maximum polynomial degree $r^{*}$. However, for the situation of just one element, it has been shown in [4] that the inf-sup constant of the element pair $\left(\mathbb{Q}_{r}, \mathbb{P}_{r-1}^{\text {disc }}\right)$ is independent of the polynomial degree $r$. Based on this result we will prove in a forthcoming paper that our global inf-sup constants $\beta\left(r^{*}\right)$ for all $r^{*}$ are bounded from below by a positive constant.

The outline of this paper is as follows: In Section 1, we introduce the notations and state the assumptions to be made on the meshes and finite element spaces. In Section 2, we introduce a new interpolation operator which is uniformly $H^{1}$-stable and divergence preserving on general meshes with hanging nodes. Based on that interpolation operator, the main result is then established in Section 3 using the macro-element technique developed by Boland and Nicolaides [6] as well as the method proposed by Fortin [12]. 


\section{Preliminaries And notation}

\subsection{General notation}

For a measurable set $G \subset \mathbb{R}^{d}$, let $(\cdot, \cdot)_{G}$ and $\|\cdot\|_{0, G}$ denote the inner product and the norm in $L^{2}(G)$ or $\left(L^{2}(G)\right)^{d}$, respectively, and let $L_{0}^{2}(G):=\left\{q \in L^{2}(G):(q, 1)_{G}=0\right\}$. Furthermore, let $|\cdot|_{m, G}$ and $\|\cdot\|_{m, G}$ denote the seminorm and norm in the Sobolev space $H^{m}(G)$ and $\left(H^{m}(G)\right)^{d}$, respectively. For a $(d-1)$-dimensional subset $E \subset \partial G$, we denote by $\langle\cdot, \cdot\rangle_{E}$ the inner product in $L^{2}(E)$, i.e.

$$
\langle u, v\rangle_{E}:=\int_{E} u(x) v(x) \mathrm{d} s .
$$

We denote by $\mathbb{P}_{m}(G)$ the space of all polynomials on the domain $G \subset \mathbb{R}^{d}$ with total degree less or equal to $m$ and by $\mathbb{Q}_{m}(G)$ the space of those polynomials where the maximum power in each coordinate is less or equal to $m$.

By $\operatorname{card}(J)$ we denote the number of elements of a finite set $J$. For a set $G \subset \mathbb{R}^{d}$, we denote by $\operatorname{int}(G)$ and $\bar{G}$ the interior and closure of $G$, respectively. Throughout this paper, $C$ will denote a generic constant which may have different values at different places whereas special constants with fixed values are indicated by $C_{1}, C_{2}, \ldots$ All these constants occurring inside of any estimates will be independent of the local and global mesh parameter $h_{K}$ and $h$, respectively, which will be defined below.

\subsection{Description of the considered grids}

Let the bounded domain $\Omega \subset \mathbb{R}^{d}$ be decomposed on a mesh $\mathcal{T}$ into elements $K \in \mathcal{T}$ which are assumed to be open quadrilaterals in the 2D-case and open hexahedrons in the 3D-case such that $\bar{\Omega}=\cup_{K \in \mathcal{T}} \bar{K}$. For an element $K \in \mathcal{T}$, we denote by $h_{K}$ the diameter of the element $K$ and by $\rho_{K}$ the diameter of the largest circle inscribed into $K$. The meshwidth $h$ of $\mathcal{T}$ is given by $h=\max _{K \in \mathcal{T}} h_{K}$.

We denote by $F_{K}: \widehat{K} \rightarrow K$ the mapping between the reference element $\widehat{K}:=(-1,+1)^{d}$ and $K$. In the sequel of this paper, the mapping $F_{K}$ is assumed to be multi-linear, i.e. $F_{K} \in\left(\mathbb{Q}_{1}(\widehat{K})\right)^{d}$. Therefore, the two dimensional faces of 3D hexahedral elements $K \in \mathcal{T}$ can be curved in general. In order to guarantee that the mapping $F_{K}$ is bijective for general non-affine quadrilateral or hexahedral meshes, the usual shape regularity assumption $h_{K} / \rho_{K} \leq C$ for all $K \in \mathcal{T}$ may not be sufficient. Therefore, we suppose the shape regularity assumptions given in $[17,19]$.

In the following, let us describe these shape regularity assumptions in more detail. By a Taylor expansion of $F_{K}(\hat{x})$ we get

$$
F_{K}(\hat{x})=b_{K}+B_{K} \hat{x}+G_{K}(\hat{x}),
$$

with $b_{K}:=F_{K}(0), B_{K}:=D F_{K}(0)$ and $G_{K}(\hat{x}):=F_{K}(\hat{x})-F_{K}(0)-D F_{K}(0)(\hat{x})$. We denote by $\widehat{S} \subset \widehat{K}$ the d-simplex with the vertices $(0, \ldots, 0),(1,0, \ldots, 0), \ldots,(0, \ldots, 0,1)$ and by $S_{K}$ the image of $\widehat{S}$ under the affine mapping $\hat{x} \rightarrow B_{K} \hat{x}+b_{K}$. For the simplices $S_{K}, K \in \mathcal{T}$, we assume the usual shape regularity assumption

$$
\frac{h_{S_{K}}}{\rho_{S_{K}}} \leq C \quad \forall K \in \mathcal{T}
$$

where $h_{S_{K}}:=\operatorname{diam}\left(S_{K}\right)$ and $\rho_{S_{K}}$ is the diameter of the largest ball inscribed into $S_{K}$. Note that condition (4) implies (see [11])

$$
\left\|B_{K}\right\| \leq C h_{S_{K}}, \quad\left\|B_{K}^{-1}\right\| \leq C h_{S_{K}}^{-1} \quad \forall K \in \mathcal{T},
$$

where $\left\|B_{K}\right\|$ denotes the matrix norm induced by the Euclidean vector norm in $\mathbb{R}^{d}$. For each element $K \in \mathcal{T}$, we define the constant $\gamma_{K}$ by

$$
\gamma_{K}:=\sup _{\hat{x} \in \widehat{K}}\left\|B_{K}^{-1} D F_{K}(\hat{x})-I\right\|,
$$


which is a measure of the deviation of $K$ from a parallelogram or a parallelepiped, respectively. Note that $\gamma_{K}=0$ if the mapping $F_{K}$ is affine.

Definition 1. A mesh $\mathcal{T}$ of quadrilateral or hexahedral elements is called shape regular if the conditions (4) and

are satisfied.

$$
\gamma_{K} \leq \gamma_{0}<1 \quad \forall K \in \mathcal{T}
$$

This shape regularity assumption imposes that the distortion of the quadrilateral or hexahedral elements from a parallelogram or parallelepiped, respectively, is uniformly bounded. These conditions guarantee further that the following properties for the mapping $F_{K}: \widehat{K} \rightarrow K$ hold (for the proof, see [17])

$$
\begin{gathered}
\left\|D F_{K}(\hat{x})\right\| \leq C h_{K} \quad \forall \hat{x} \in \widehat{K}, \\
C h_{K}^{d} \leq\left|\operatorname{det}\left(D F_{K}(\hat{x})\right)\right| \leq C^{\prime} h_{K}^{d} \quad \forall \hat{x} \in \widehat{K} .
\end{gathered}
$$

In this paper, our main interest is to study the effect on the inf-sup condition of using hanging nodes. That means that the usual assumption of a regular grid $\mathcal{T}$ has to be weakened. In the following, we will describe the type of grids that is treated in this paper. $\mathcal{T}$ is a multi-level grid generated by a refinement process in the following way. We start with a partition $\mathcal{T}^{0}$ of the domain $\Omega$ into elements $K \in \mathcal{T}^{0}$ of grid level 0, i.e. $\Omega=\operatorname{int}\left(\bigcup_{K \in \mathcal{T}^{0}} \bar{K}\right)$. The grid $\mathcal{T}^{0}$ is assumed to be regular in the usual sense, i.e. for any two different elements $K_{1}, K_{2} \in \mathcal{T}^{0}$ the intersection $\overline{K_{1}} \cap \overline{K_{2}}$ is either empty or a common $(d-m)$-dimensional face of $K_{1}$ and $K_{2}$ where $m \in\{1, \ldots, d\}$. The set of the neighboring elements of the element $K$ is denoted by

$$
\Lambda(K):=\{\widetilde{K} \in \mathcal{T}: \bar{K} \cap \overline{\widetilde{K}} \neq \emptyset\} .
$$

Now, starting with the elements $K \in \mathcal{T}^{0}$, an existing element $K$ can be refined, i.e. it can be splitted into $2^{d}$ many new elements called son-elements and denoted by $\sigma_{i}(K), i=1, \ldots, 2^{d}$ (see Fig. 2). For a new element $K^{\prime}=\sigma_{i}(K)$, we will say that $K$ is the father-element of $K^{\prime}$ and we will write $K=\mathcal{F}\left(K^{\prime}\right)$. If an element $K$ is refined then, in the partition of the domain $\Omega$, it is replaced by the set of its son-elements $\sigma_{i}(K), i=1, \ldots, 2^{d}$. The new elements can be refined again and again and so the final partition $\mathcal{T}$ of $\Omega$ is created.

Definition 2. For an element $K \in \mathcal{T}$, generated by the refinement process from the initial grid $\mathcal{T}^{0}$, we define the refinement level $\ell(K)$ as $\ell(K):=0$ if $K \in \mathcal{T}^{0}$ and $\ell(K):=m \geq 1$ if there exists a chain of $m$ fatherelements $K_{i}, i=1, \ldots, m$, starting from $K_{0}:=K$ and defined by $K_{i}:=\mathcal{F}\left(K_{i-1}\right)$ for $i=1, \ldots, m$, such that $K_{m} \in \mathcal{T}^{0}$.

The above defined refinement level $\ell(K)$ is equal to the number of refinement steps that is needed to generate element $K$ from an element of the coarsest grid $\mathcal{T}^{0}$.

Definition 3. A grid $\mathcal{T}$, generated by the refinement process from the initial grid $\mathcal{T}^{0}$, is called 1-irregular if

$$
\left|\ell(K)-\ell\left(K^{\prime}\right)\right| \leq 1
$$

for any pair of neighbored elements $K, K^{\prime} \in \mathcal{T}$ where $\partial K \cap \partial K^{\prime}$ is a one- or two-dimensional manifold.

In this paper, we consider only grids $\mathcal{T}$ which are 1-irregular.

Finally, we need some additional notation for the analysis in the following sections. We denote by $\mathcal{E}(K)$ the set of all $(d-1)$-dimensional faces of an element $K$, by $n^{K}$ the unit normal vector on the element boundary $\partial K$ directed outward with respect $K$ and by $n_{E}^{K}$ the restriction of the normal vector $n^{K}$ to the face $E \in \mathcal{E}(K)$. Let $\mathcal{E}$ be the set of all faces $E \in \mathcal{E}(K)$ of all elements $K \in \mathcal{T}$. We split $\mathcal{E}$ in the form

$$
\mathcal{E}=\mathcal{E}_{0} \cup \mathcal{E}(\Gamma)
$$




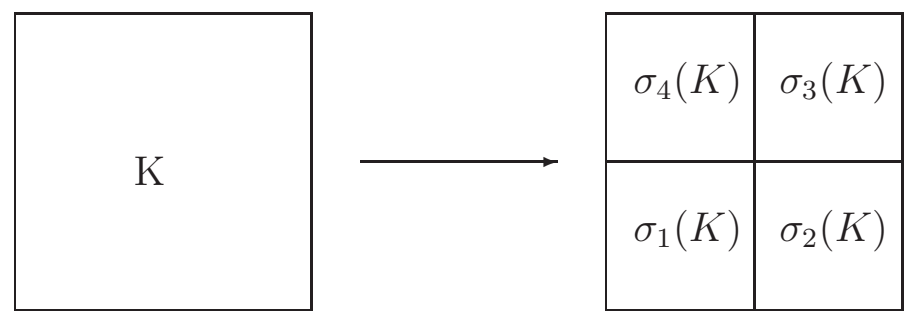

Figure 2. Refinement of element $K$ into son-elements $\sigma_{i}(K), i=1, \ldots, 4$.
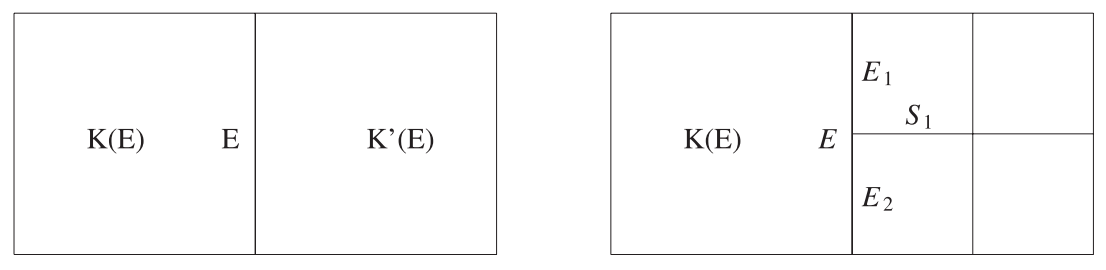

Figure 3. Two dimensional configuration for the case of a regular inner face i.e. $E \in \mathcal{E}_{r}$ (left) and for the case of an irregular inner face i.e. $E \in \mathcal{E}_{i}$ (right).

where $\mathcal{E}_{0}$ denotes the set of all inner faces of $\mathcal{E}$ and $\mathcal{E}(\Gamma)$ the set of all faces of $\mathcal{E}$ located at the boundary $\Gamma$ of $\Omega$. For any face $E \in \mathcal{E}$, we associate the set $\mathcal{T}(E)$ of adjacent elements defined by

$$
\mathcal{T}(E):=\{K \in \mathcal{T}: E \in \mathcal{E}(K)\}
$$

Let $\mathcal{E}_{r}$ denote the set of the regular inner faces defined as

$$
\mathcal{E}_{r}:=\left\{E \in \mathcal{E}_{0}: \operatorname{card}(\mathcal{T}(E))=2\right\}
$$

For each regular face $E \in \mathcal{E}_{r}$, there exist exactly two different elements denoted by $K(E)$ and $K^{\prime}(E)$ such that $E$ is one of their faces (see Fig. 3), i.e.

$$
\mathcal{T}(E)=\left\{K(E), K^{\prime}(E)\right\} \quad \forall E \in \mathcal{E}_{r}
$$

For all other faces $E \in \mathcal{E} \backslash \mathcal{E}_{r}$, there is only one element denoted by $K(E)$ which has $E$ as one of its faces, i.e.

$$
\mathcal{T}(E)=\{K(E)\} \quad \forall E \in \mathcal{E} \backslash \mathcal{E}_{r}
$$

A face $\widetilde{E} \in \mathcal{E}$ is called a son-face of a face $E \in \mathcal{E}$ if $\widetilde{E} \subset E$ and $|\widetilde{E}|<|E|$ where $|\widetilde{E}|$ and $|E|$ denote the $(d-1)$-dimensional measure of $\widetilde{E}$ and $E$, respectively. We denote by $\sigma(E)$ the set of all son-faces of $E$. In Figure 3 (right part) we have e.g. $\sigma(E)=\left\{E_{1}, E_{2}\right\}$. Note that for each regular face $E \in \mathcal{E}_{r}$, the set $\sigma(E)$ is empty. We denote by $\mathcal{E}_{i}$ the set of all irregular inner faces defined as

$$
\mathcal{E}_{i}:=\left\{E \in \mathcal{E}_{0}: \sigma(E) \neq \emptyset\right\}
$$

Using these definitions, the set $\mathcal{E}_{0}$ of all inner faces can be decomposed as

$$
\mathcal{E}_{0}=\mathcal{E}_{r} \cup \mathcal{E}_{i} \cup\left(\bigcup_{E \in \mathcal{E}_{i}} \sigma(E)\right)
$$


Let $\widetilde{E} \in \sigma(E)$ be a son-face of $E \in \mathcal{E}_{i}$, then the face $E$ is called the father-face of $\widetilde{E}$ and we write $E=\mathcal{F}(\widetilde{E})$. We define the set of all son-faces by

$$
\mathcal{E}_{\sigma}:=\bigcup_{E \in \mathcal{E}_{i}} \sigma(E)
$$

For a given element $K \in \mathcal{T}$, we define further

$$
\mathcal{E}_{\mu}(K):=\mathcal{E}(K) \cap \mathcal{E}_{\mu} \quad \text { for } \mu \in\{r, i, \sigma\} .
$$

Finally, for each face $E \in \mathcal{E}$, let $n_{E}$ denote the unit vector $n_{E}^{K(E)}$ which is normal to the face $E$ and directed outward with respect to the element $K(E)$.

As a consequence of the shape regularity of the mesh we have

$$
|E| \leq C_{5} h_{K}^{d-1} \quad \forall E \in \mathcal{E}(K)
$$

where $|E|$ describes the $(d-1)$ dimensional measure of $E$ (see [17], Lem. 5). Furthermore, the following inequality will be needed (see [17], Lem. 4)

$$
C_{6} h_{\widetilde{K}} \leq h_{K} \leq C_{7} h_{\widetilde{K}} \quad \forall \widetilde{K} \in \Lambda(K) .
$$

\subsection{Finite element spaces}

Let $\mathbf{r}$ denote the degree vector $\mathbf{r}:=\left\{r_{K}: K \in \mathcal{T}\right\}$ which contains the polynomial degree $r_{K}$ of the velocity approximation on each element $K$. In this paper, we assume that

$$
r_{K} \geq 2 \quad \forall K \in \mathcal{T} .
$$

The finite element space of the velocity $X^{N} \subset\left(H_{0}^{1}(\Omega)\right)^{d}$ associated with the mesh $\mathcal{T}$ and the degree vector $\mathbf{r}$ is defined as $X^{N}:=\left(S^{N}\right)^{d}$ with the scalar finite element space

$$
\begin{aligned}
S^{N} & :=S(\Omega ; \mathbf{r}, \mathcal{T}) \\
& :=\left\{\phi \in H_{0}^{1}(\Omega):\left.\phi\right|_{K} \circ F_{K} \in \mathbb{Q}_{r_{K}}(\widehat{K}) \quad \forall K \in \mathcal{T}\right\} .
\end{aligned}
$$

The finite element space $M^{N} \subset L_{0}^{2}(\Omega)$ for the approximation of the pressure is chosen as the following mapped space

$$
\begin{aligned}
M^{N} & :=M(\Omega ; \mathbf{r}, \mathcal{T}) \\
& :=\left\{q \in L_{0}^{2}(\Omega):\left.q\right|_{K} \circ F_{K} \in \mathbb{P}_{r_{K}-1}(\hat{K}) \quad \forall K \in \mathcal{T}\right\} .
\end{aligned}
$$

Then, the discrete Stokes problem reads: Find $\left(u^{N}, p^{N}\right) \in X^{N} \times M^{N}$ such that

$$
\begin{aligned}
\nu\left(\nabla u^{N}, \nabla v^{N}\right)_{\Omega}-\left(p^{N}, \nabla \cdot v^{N}\right)_{\Omega} & =\left(f, v^{N}\right)_{\Omega} & & \forall v^{N} \in X^{N} \\
\left(q^{N}, \nabla \cdot u^{N}\right)_{\Omega} & =0 & & \forall q^{N} \in M^{N} .
\end{aligned}
$$

Remark 4. Instead of the mapped pressure space $M^{N}$ we could also choose, like in [13], Section II.3.2, the unmapped space $\widetilde{M}^{N}$ defined by

$$
\widetilde{M}^{N}:=\left\{q \in L_{0}^{2}(\Omega):\left.q\right|_{K} \in \mathbb{P}_{r_{K}-1}(K) \quad \forall K \in \mathcal{T}\right\} .
$$

Concerning the implementation, this space would be less attractive than the mapped space $M^{N}$ defined in (15). However, in contrast to the mapped version, the unmapped pressure space guarantees optimal approximation 
properties on general quadrilateral or hexahedral meshes [5]. In the following we will consider only the case of a mapped pressure space. However, the presented theory can also be applied to the case of an unmapped space (see Rem. 16 below).

For the subsequent analysis we need also the finite element spaces for the velocity with the polynomial order of 1 and 2, i.e. $X_{h}^{1}:=\left(S_{h}^{1}\right)^{d}$ and $X_{h}^{2}:=\left(S_{h}^{2}\right)^{d}$ based on the scalar finite element spaces

$$
S_{h}^{r}:=\left\{\phi \in H_{0}^{1}(\Omega):\left.\phi\right|_{K} \circ F_{K} \in \mathbb{Q}_{r}(\widehat{K}) \forall K \in \mathcal{T}\right\}, \quad r \in\{1,2\}
$$

as well as the finite element space of the piecewise constant pressure functions

$$
M_{h}^{0}:=\left\{q \in L_{0}^{2}(\Omega):\left.q\right|_{K} \in \mathbb{P}_{0}(K) \quad \forall K \in \mathcal{T}\right\} .
$$

Some attention is required to ensure interelement continuity in (14) and (18) in the case of hanging nodes or if $r_{K}$ is variable on the adjacent cells $K \in \mathcal{T}(E)$ of an inner face $E$. We refer to [21] for a detailed treatment of this issue.

\section{TWO INTERPOLATION OPERATORS}

In order to prove the inf-sup condition (2) for the finite element pairs $\left(X^{N}, M^{N}\right)$ on locally refined grids with hanging nodes we will use the macro-element technique proposed by Boland and Nicolaides [6]. This technique requires a local inf-sup condition with a uniform constant for all subdomains of a nonoverlapping partitioning of $\Omega$ and a global inf-sup condition for a pair of subspaces $\left(\bar{X}^{N}, M_{h}^{0}\right)$ where $\bar{X}^{N}$ is a suitable subspace of $X^{N}$ and $M_{h}^{0}$ is the space of discontinuous piecewise constant pressure functions. As the subdomains we choose the grid cells $K \in \mathcal{T}$. Then, the local inf-sup condition can be adopted from the literature (see Sect. 3 below) and the only thing which is still to do is to prove a global inf-sup condition for a suitable pair of spaces $\left(\bar{X}^{N}, M_{h}^{0}\right)$.

The goal of this section is to derive the tools that are needed to prove this condition for the low order pair $\left(X_{h}^{2}, M_{h}^{0}\right)$. To this end, we follow the method proposed by Fortin [12] and construct an interpolation operator $\Pi_{h}:\left(H_{0}^{1}(\Omega)\right)^{d} \rightarrow X_{h}^{2}$ which is $H^{1}$-stable uniformly with respect to $h$, i.e.

$$
\left|\Pi_{h} v\right|_{1, \Omega} \leq C|v|_{1, \Omega} \quad \forall v \in\left(H_{0}^{1}(\Omega)\right)^{d},
$$

and divergence preserving with respect to the test space $M_{h}^{0}$, i.e.

$$
\left(\operatorname{div} \Pi_{h} v, q\right)=(\operatorname{div} v, q) \quad \forall q \in M_{h}^{0} .
$$

In our approach, the interpolation operator $\Pi_{h}$ is defined as

$$
\Pi_{h} v:=R_{h} v+I_{h}\left(v-R_{h} v\right) \quad \forall v \in\left(H_{0}^{1}(\Omega)\right)^{d},
$$

with a divergence preserving operator $I_{h}$ presented in Section 2.1 and the Scott-Zhang type interpolation operator $R_{h}$ proposed in [17].

\subsection{Divergence preserving operator $I_{h}$}

The operator $I_{h}:\left(H_{0}^{1}(\Omega)\right)^{d} \rightarrow X_{h}^{2}$ is assumed to have the following form

$$
I_{h} v=\sum_{E \in \mathcal{E}_{r} \cup \mathcal{E}_{i}} I_{h}^{E} v \quad \forall v \in\left(H_{0}^{1}(\Omega)\right)^{d}
$$

where the construction of the local operators $I_{h}^{E}$ depends on the dimension $d$ of the considered problem as well as on whether $E \in \mathcal{E}_{r}$ or $E \in \mathcal{E}_{i}$. 
At first, let us describe those parts of the construction of $I_{h}^{E}$ which can be presented in a general fashion for both the two and three-dimensional case. Let $E \in \mathcal{E}_{r} \cup \mathcal{E}_{i}$ be a given face, $K=K(E)$ the element associated with $E$ as defined in Section 2.2 and $F_{K}: \widehat{K} \rightarrow K$ the corresponding mapping between the reference element $\widehat{K}=(-1,1)^{d}$ and $K$. Then, by $\widehat{E}$ we denote the face of the reference element $\widehat{K}$ that corresponds to $E$ in the sense that $\widehat{E}=F_{K}^{-1}(E)$. Let $\widehat{m}_{\widehat{E}}$ be the barycenter of the reference face $\widehat{E}$ and $m_{E}:=F_{K}\left(\widehat{m}_{\widehat{E}}\right)$ the corresponding point at the original face $E$. Then, the face bubble function $\Phi_{E} \in X_{h}^{2}$ is defined as

$$
\Phi_{E}(x):=\psi_{E}(x) n_{E}^{0} \quad \forall x \in \Omega,
$$

where $n_{E}^{0}:=n_{E}\left(m_{E}\right)$ is the unit normal vector $n_{E}$ at the point $m_{E} \in E$ and $\psi_{E} \in S_{h}^{2}$ denotes the scalar function defined by its nodal values in the following way.

Let $a_{j} \in \Omega, j \in J^{2}$, denote the nodal points of the quadratic finite element space $S_{h}^{2}$. These nodal points are the images of the reference nodes $\widehat{a}_{m} \in \widehat{K}, m=1, \ldots, 3^{d}$, under the reference mappings $F_{K}: \widehat{K} \rightarrow K$ of all elements $K \in \mathcal{T}$. The reference nodes $\widehat{a}_{m}$ are the vertices of $\widehat{K}$, the barycenter of $\widehat{K}$, barycenters of the $(d-1)$-dimensional faces of $\widehat{K}$ and, in the three dimensional case, additionally the midpoints of the edges of $\widehat{K}$. For a given element $K \in \mathcal{T}$, we define by

$$
J^{2}(K):=\left\{j \in J^{2}: a_{j}=F_{K}\left(\widehat{a}_{m}\right), 1 \leq m \leq 3^{d}\right\}
$$

the local index set of the nodal points $a_{j}$ that belong to element $K$. Note that among the nodal points $a_{j} \in \Omega$, $j \in J^{2}$, there are also the so called hanging nodes. A node $j \in J^{2}$ is called a hanging node if there exists an irregular face $E \in \mathcal{E}_{i}$ and a son-face $\widetilde{E} \in \sigma(E)$ such that $j \in J^{2}(K(\widetilde{E}))$ but $j \notin J^{2}(K(E))$ where $K(\widetilde{E})$ and $K(E)$ denote the uniquely determined elements that are assigned to the faces $\widetilde{E}$ and $E$, respectively, as defined in Section 2.2. A node $j \in J^{2}$ is called a regular node if it is not a hanging node. In the following, the set of all regular nodes will be denoted by $J_{r}^{2}$. Now, a finite element function $\psi \in S_{h}^{2}$ is uniquely determined by its nodal values $\psi\left(a_{j}\right)$ for all regular nodes $j \in J_{r}^{2}$. The values $\psi\left(a_{j}\right)$ for hanging nodes are determined by the continuity requirements of $\psi \in S_{h}^{2}$. Note that for all regular and irregular faces $E$, the barycenter $m_{E}$ of $E$ corresponds to a regular node. Thus, our scalar face bubble function $\psi_{E} \in S_{h}^{2}$ associated with the face $E \in \mathcal{E}_{r} \cup \mathcal{E}_{i}$ is defined by

$$
\psi_{E}\left(a_{j}\right):= \begin{cases}0 & \text { for all } j \in J_{r}^{2} \text { with } a_{j} \neq m_{E} \\ 1 & \text { if } a_{j}=m_{E}\end{cases}
$$

For the support of $\Phi_{E}$, we have (see Fig. 3 for the 2D-case)

$$
\operatorname{supp}\left(\Phi_{E}\right)=\overline{K(E) \cup K^{\prime}(E)} \quad \forall E \in \mathcal{E}_{r}
$$

and

$$
\operatorname{supp}\left(\Phi_{E}\right)=\overline{K(E)} \cup \overline{\bigcup_{\widetilde{E} \in \sigma(E)} K(\widetilde{E})} \quad \forall E \in \mathcal{E}_{i} .
$$

From the definition of $\Phi_{E} \in X_{h}^{2}$ we immediately get

$$
\max _{x \in \bar{\Omega}}\left\|\Phi_{E}(x)\right\| \leq C_{1} \quad \forall E \in \mathcal{E}_{r} \cup \mathcal{E}_{i}
$$

which implies by means of inverse inequalities the estimate

$$
\left|\Phi_{E}\right|_{1, K} \leq C_{2} h_{K}^{d / 2-1} \quad \forall K \in \mathcal{T} .
$$

The constants $C_{1}$ and $C_{2}$ are independent of $E$ and $K$ respectively. 
Lemma 5. Let $\mathcal{T}$ be a shape-regular and 1-irregular mesh. Let $E \in \mathcal{E}_{r} \cup \mathcal{E}_{i}$ and $K \in \mathcal{T}$ such that $E \in \mathcal{E}(K)$. Then, there exist positive constants $C_{3}$ and $C_{4}$ independent of $E$ and $K$ such that

$$
\left\langle\Phi_{E} \cdot n_{E}, 1\right\rangle_{E} \geq C_{3} h_{K}^{d-1}
$$

and

$$
\|v\|_{L^{2}(E)} \leq C_{4} h_{K}^{1 / 2}\left\{h_{K}^{-1}\|v\|_{0, K}+|v|_{1, K}\right\} \quad \forall v \in\left(H^{1}(K)\right)^{d} .
$$

Proof. First, we prove the inequality (28).

For the two dimensional case $d=2$, the unit normal vector $n_{E}$ is constant on $E$, i.e. $n_{E}(x)=n_{E}^{0}$ for all points $x \in E$. By means of the Simpson's rule, we therefore have

$$
\left\langle\Phi_{E} \cdot n_{E}, 1\right\rangle_{E}=\left\langle\psi_{E}, 1\right\rangle_{E}=\frac{4}{6}|E| \geq C_{3} h_{K}
$$

where $|E|$ denotes the length of the face $E$ and the last inequality follows from the shape regularity of the mesh.

For the three dimensional case $d=3$, the proof is slightly more involved since $n_{E}(x)$ may not be constant any more for $x \in E$. We use the mapping $F_{K}: \widehat{K} \rightarrow K$ between the reference element $\widehat{K}=(-1,1)^{3}$ and the element $K$ in order to get a parametrization of the face $E \in \mathcal{E}(K)$. We denote by $\widehat{E}=F_{K}^{-1}(E)$ the face of $\widehat{K}$ that corresponds to $E$. First, we assume the case where $\widehat{E}$ is the face defined by $\hat{x}_{1}=-1$. Then, we can represent each point $\hat{x} \in \widehat{E}$ as

$$
\hat{x}=\widehat{\gamma}\left(t_{1}, t_{2}\right):=\left(-1, t_{1}, t_{2}\right)^{T} \quad \text { where }\left(t_{1}, t_{2}\right) \in G:=(-1,1)^{2},
$$

and each point $x \in E$ as

$$
x=\gamma\left(t_{1}, t_{2}\right):=F_{K}\left(\widehat{\gamma}\left(t_{1}, t_{2}\right)\right), \quad \forall\left(t_{1}, t_{2}\right) \in G .
$$

Then, the vector $N_{E}$ defined as

$$
N_{E}\left(t_{1}, t_{2}\right)=\left.\left(\frac{\partial \gamma}{\partial t_{1}} \times \frac{\partial \gamma}{\partial t_{2}}\right)\right|_{\left(t_{1}, t_{2}\right)}
$$

is a normal vector of $E$ at the point $x=\gamma\left(t_{1}, t_{2}\right)$. Furthermore, there is constant sign factor $s_{E} \in\{-1,+1\}$ such that the normal unit vector $n_{E}$ can be uniquely expressed as

$$
n_{E}(x)=n_{E}\left(\gamma\left(t_{1}, t_{2}\right)\right)=\frac{s_{E}}{\left\|N_{E}\left(t_{1}, t_{2}\right)\right\|} N_{E}\left(t_{1}, t_{2}\right) \quad \forall\left(t_{1}, t_{2}\right) \in G,
$$

under the constraint $n_{E}(\gamma(0,0))=n_{E}^{0}$. Then, we obtain

$$
\left\langle\Phi_{E} \cdot n_{E}, 1\right\rangle_{E}=\int_{G} \psi_{E}\left(\gamma\left(t_{1}, t_{2}\right)\right) \frac{N_{E}(0,0) \cdot N_{E}\left(t_{1}, t_{2}\right)}{\left\|N_{E}(0,0)\right\|} \mathrm{d} t_{1} \mathrm{~d} t_{2} .
$$

A simple calculation shows that the integrand in $(30)$ is a polynomial in the space $\mathbb{Q}_{3}(G)$, such that Simpson's rule yields the exact value of the integral, i.e.

$$
\left\langle\Phi_{E} \cdot n_{E}, 1\right\rangle_{E}=\frac{16}{9} \psi_{E}(\gamma(0,0)) \cdot\left\|N_{E}(0,0)\right\|=\frac{16}{9}\left\|N_{E}(0,0)\right\|
$$

Now, we have

$$
\begin{aligned}
\operatorname{det}\left(D F_{K}(\widehat{\gamma}(0,0))\right) & =\left.\frac{\partial F_{K}}{\partial \hat{x}_{1}} \cdot\left(\frac{\partial F_{K}}{\partial \hat{x}_{2}} \times \frac{\partial F_{K}}{\partial \hat{x}_{3}}\right)\right|_{\hat{x}=\widehat{\gamma}(0,0)} \\
& =\left.\frac{\partial F_{K}}{\partial \hat{x}_{1}}\right|_{\hat{x}=\widehat{\gamma}(0,0)} \cdot N_{E}(0,0),
\end{aligned}
$$


which implies the estimate

$$
\left|\operatorname{det}\left(D F_{K}(\widehat{\gamma}(0,0))\right)\right| \leq\left\|\frac{\partial F_{K}}{\partial \hat{x}_{1}}(\widehat{\gamma}(0,0))\right\| \cdot\left\|N_{E}(0,0)\right\| .
$$

Using the estimates (9) and (8), this leads to

$$
\left\|N_{E}(0,0)\right\| \geq C h_{K}^{2}
$$

Together with (31) this completes the proof of (28) in the case that $\widehat{E}$ corresponds to $\hat{x}_{1}=-1$. The proof where $\widehat{E}$ corresponds to $\hat{x}_{i}= \pm 1$ is completely analogous.

Now, we prove the estimate (29). At first, we need to prove the estimate

$$
\|v\|_{L^{2}(E)} \leq C h_{K}^{(d-1) / 2}\|\hat{v}\|_{L^{2}(\widehat{E})}
$$

where the function $\hat{v} \in\left(H^{1}(\widehat{K})\right)^{d}$ is defined by $\hat{v}:=v\left(F_{K}(\hat{x})\right)$ for all $\hat{x} \in \widehat{K}$. We present the proof of (32) only for the three dimensional case $d=3$. The case $d=2$ follows easily by simple transformation of the integrals corresponding to both sides of (32).

Again we consider only the special case where $\widehat{E}=F_{K}^{-1}(E)$ corresponds to $\hat{x}_{1}=-1$. For the face $E$, we get

$$
\|v\|_{L^{2}(E)}^{2}=\int_{G} v\left(\gamma\left(t_{1}, t_{2}\right)\right)^{2}\left\|N_{E}\left(t_{1}, t_{2}\right)\right\| \mathrm{d} t_{1} \mathrm{~d} t_{2}
$$

and by means of (8)

which implies

$$
\left\|N_{E}\left(t_{1}, t_{2}\right)\right\| \leq\left\|\frac{\partial F_{K}}{\partial \hat{x}_{2}}\left(\widehat{\gamma}\left(t_{1}, t_{2}\right)\right)\right\| \cdot\left\|\frac{\partial F_{K}}{\partial \hat{x}_{3}}\left(\widehat{\gamma}\left(t_{1}, t_{2}\right)\right)\right\| \leq C h_{K}^{2},
$$

$$
\|v\|_{L^{2}(E)}^{2} \leq C h_{K}^{2} \int_{G} v\left(\gamma\left(t_{1}, t_{2}\right)\right)^{2} \mathrm{~d} t_{1} \mathrm{~d} t_{2} .
$$

The parametrization of $\widehat{E}$ is given by $\hat{x}=\widehat{\gamma}\left(t_{1}, t_{2}\right)=\left(-1, t_{1}, t_{2}\right)^{T}$ for $\left(t_{1}, t_{2}\right) \in G$ and the corresponding normal vector is

Therefore, we get

$$
\widehat{N}_{\widehat{E}}\left(t_{1}, t_{2}\right):=\left.\left(\frac{\partial \widehat{\gamma}}{\partial t_{1}} \times \frac{\partial \widehat{\gamma}}{\partial t_{2}}\right)\right|_{\left(t_{1}, t_{2}\right)}=(1,0,0)^{T}
$$

$$
\begin{aligned}
\|\hat{v}\|_{L^{2}(\widehat{E})}^{2} & =\int_{G} \hat{v}\left(\widehat{\gamma}\left(t_{1}, t_{2}\right)\right)^{2}\left\|\widehat{N}_{\widehat{E}}\left(t_{1}, t_{2}\right)\right\| \mathrm{d} t_{1} \mathrm{~d} t_{2} \\
& =\int_{G} v\left(\gamma\left(t_{1}, t_{2}\right)\right)^{2} \mathrm{~d} t_{1} \mathrm{~d} t_{2} .
\end{aligned}
$$

Together with (33), this proves (32) for the case $d=3$. Now, we apply the trace theorem on the reference element $\widehat{K}$ and well-known estimates between the norms of $\hat{v}$ and $\widehat{K}$ and the norms of $v$ on $K$ (see e.g. [11]) and get

$$
\|\hat{v}\|_{L^{2}(\widehat{E})} \leq C\|\hat{v}\|_{0, \widehat{K}}+C|\hat{v}|_{1, \widehat{K}} \leq C h_{K}^{-d / 2}\|v\|_{0, K}+C h_{K}^{1-d / 2}|v|_{1, K} .
$$

Together with (32), this proves the estimate (29).

In case of a regular face $E \in \mathcal{E}_{r}$, we impose for an arbitrary function $v \in\left(H_{0}^{1}(\Omega)\right)^{d}$

$$
I_{h}^{E} v=c_{E}(v) \Phi_{E}
$$


where $c_{E}(v) \in \mathbb{R}$ is a constant which is chosen such that

$$
c_{E}(v)\left\langle\Phi_{E} \cdot n_{E}, 1\right\rangle_{E}=\left\langle v \cdot n_{E}, 1\right\rangle_{E}
$$

Due to (28) the term $\left\langle\Phi_{E} \cdot n_{E}^{K}, 1\right\rangle_{E}$ does not vanish. By definition of $\Phi_{E}$ it holds $\Phi_{E}=0$ on $\partial K \backslash E$ for all $K \in \mathcal{T}(E)$. Thus, the operator $I_{h}^{E}$ has the property that for each function $v \in\left(H_{0}^{1}(\Omega)\right)^{d}$ it holds

$$
\left\langle I_{h}^{E} v \cdot n^{K}, 1\right\rangle_{\partial K}=\left\langle v \cdot n_{E}^{K}, 1\right\rangle_{E},
$$

for all $E \in \mathcal{E}_{r}$ and for all $K \in \mathcal{T}(E)=\left\{K(E), K^{\prime}(E)\right\}$.

In the following, we will describe those parts of the construction of $I_{h}^{E}$ which are different in the two and three-dimensional case.

\subsubsection{Irregular faces in the two dimensional case}

In case of $E \in \mathcal{E}_{i}$, we denote by $E_{1}$ and $E_{2}$ the son-faces of $E$ and by $K_{m}:=K\left(E_{m}\right), m \in\{1,2\}$, the element associated with the face $E_{m}$ as depicted in Figure 3 (right part). Moreover, we denote by $S_{1}:=\overline{K_{1}} \cap \overline{K_{2}}$ the common face of the elements $K_{1}$ and $K_{2}$. Then, we impose

$$
I_{h}^{E} v:=c_{E}(v) \Phi_{E}+c_{E, S_{1}}(v) \Phi_{S_{1}}
$$

where $c_{E}(v)$ and $c_{E, S_{1}}(v)$ are suitable constants. The constant $c_{E}(v)$ again is chosen such that equation (35) is satisfied which is always possible due to the fact that $\left\langle\Phi_{E} \cdot n_{E}, 1\right\rangle_{E} \neq 0$. Since the face-bubble function $\Phi_{S_{1}}$ is zero on the face $E$ and $\Phi_{E}=0$ on $\partial K(E) \backslash E$, the operator $I_{h}^{E}$ has the following property for all functions $v \in\left(H_{0}^{1}(\Omega)\right)^{d}$

$$
\left\langle I_{h}^{E} v \cdot n^{K(E)}, 1\right\rangle_{\partial K(E)}=\left\langle v \cdot n_{E}^{K(E)}, 1\right\rangle_{E},
$$

for all $E \in \mathcal{E}_{i}$. The constant $c_{E, S_{1}}(v)$ will be taken such that the operator $I_{h}^{E}$ satisfies the following condition

$$
\left\langle I_{h}^{E} v \cdot n^{K(\widetilde{E})}, 1\right\rangle_{\partial K(\widetilde{E})}=\left\langle v \cdot n_{\widetilde{E}}^{K(\widetilde{E})}, 1\right\rangle_{\widetilde{E}},
$$

for all $E \in \mathcal{E}_{i}$ and for all $\widetilde{E} \in \sigma(E)$. A simple calculation starting from equation (39) with $\widetilde{E}=E_{i}$ leads to the expression

$$
c_{E, S_{1}}(v)=\left\langle\Phi_{S_{1}} \cdot n_{S_{1}}^{K_{i}}, 1\right\rangle_{S_{1}}^{-1}\left\{\left\langle v \cdot n_{E_{i}}^{K_{i}}, 1\right\rangle_{E_{i}}-c_{E}(v)\left(\left\langle\Phi_{E} \cdot n_{E_{i}}^{K_{i}}, 1\right\rangle_{E_{i}}+\left\langle\Phi_{E} \cdot n_{S_{1}}^{K_{i}}, 1\right\rangle_{S_{1}}\right)\right\} .
$$

It is easy to show that the value on the right hand side is the same for $i \in\{1,2\}$. The expression for $c_{E, S_{1}}(v)$ in (40) is well defined since by (28) it holds $\left|\left\langle\Phi_{S_{1}} \cdot n_{S_{1}}^{K_{1}}, 1\right\rangle_{S_{1}}\right| \geq C h_{K_{1}}$. Using this estimate, (26) and the estimate (12) between $h_{K_{1}}$ and $h_{K}$ with $K:=K(E)$, we obtain from (40) with $i=1$

$$
\begin{aligned}
\left|c_{E, S_{1}}(v)\right| & \leq C h_{K_{1}}^{-1}\left\{\left|E_{1}\right|^{1 / 2}\|v\|_{L^{2}\left(E_{1}\right)}+\left|c_{E}(v)\right| \max _{x \in \bar{\Omega}}\left\|\Phi_{E}(x)\right\|\left(\left|E_{1}\right|+\left|S_{1}\right|\right)\right\} \\
& \leq C h_{K}^{-1 / 2}\|v\|_{L^{2}(E)}+C\left|c_{E}(v)\right| .
\end{aligned}
$$

\subsubsection{Irregular faces in the three dimensional case}

In case of $E \in \mathcal{E}_{i}$, we will denote by $E_{m}, m=1, \ldots, 4$, the son-faces of $E$ and by $K_{m}:=K\left(E_{m}\right)$ the element associated with the face $E_{m}$ as depicted in Figure 4 . Furthermore, we define $S_{m}:=\overline{K_{m}} \cap \overline{K_{\widetilde{m}}}$ as the common 


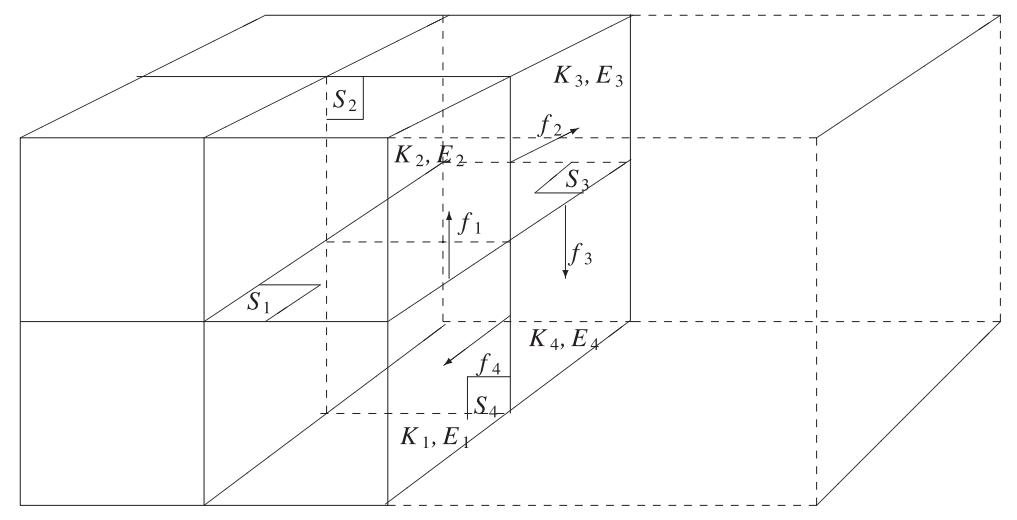

Figure 4. Three dimensional configuration for the case of an irregular inner face i.e. $E \in \mathcal{E}_{i}$.

face of the elements $K_{m}$ and $K_{\widetilde{m}}$ where $\widetilde{m}:=m+1$ if $m \in\{1,2,3\}$ and $\widetilde{m}:=1$ if $m=4$. Now, we impose

$$
I_{h}^{E} v:=c_{E}(v) \Phi_{E}+\sum_{m=1}^{4} c_{E, S_{m}}(v) \Phi_{S_{m}}
$$

where $c_{E}(v)$ and $\left\{c_{E, S_{m}}(v)\right\}_{1 \leq m \leq 4}$ are suitable constants. For a given function $v \in\left(H_{0}^{1}(\Omega)\right)^{d}$, the constant $c_{E}(v)$ is chosen such that equation (35) is satisfied which is always possible due to the fact that $\left\langle\Phi_{E} \cdot n_{E}, 1\right\rangle_{E} \neq 0$. Since the face-bubble functions $\left\{\Phi_{S_{m}}\right\}_{1 \leq m \leq 4}$ are zero on the face $E$ and $\Phi_{E}=0$ on $\partial K(E) \backslash E$, the operator $I_{h}^{E}$ has the same property as in the 2D-case that for all functions $v \in\left(H_{0}^{1}(\Omega)\right)^{d}$

$$
\left\langle I_{h}^{E} v \cdot n^{K(E)}, 1\right\rangle_{\partial K(E)}=\left\langle v \cdot n_{E}^{K(E)}, 1\right\rangle_{E},
$$

for all $E \in \mathcal{E}_{i}$.

Lemma 6. Let $\mathcal{T}$ be a shape-regular and 1-irregular mesh. For a face $E \in \mathcal{E}_{i}$, let $K=K(E)$ and $I_{h}^{E} v$ be the local interpolation defined in (42). Then, for any given constant $c_{E}(v)$ there exist constants $\left\{c_{E, S_{m}}(v)\right\}_{1 \leq m \leq 4}$ such that

$$
\left\langle I_{h}^{E} v \cdot n^{K(\widetilde{E})}, 1\right\rangle_{\partial K(\widetilde{E})}=\left\langle v \cdot n_{\widetilde{E}}^{K(\widetilde{E})}, 1\right\rangle_{\widetilde{E}} \quad \forall \widetilde{E} \in \sigma(E)
$$

and

$$
\left|c_{E, S_{m}}(v)\right| \leq C_{8} h_{K}^{-1}\|v\|_{L^{2}(E)}+C_{9}\left|c_{E}(v)\right| \quad \forall m=1, \ldots, 4,
$$

where the constants $C_{8}$ and $C_{9}$ do not depend on $E, h_{K}$ and the function $v \in\left(H_{0}^{1}(\Omega)\right)^{3}$.

Proof. In the following, we will consider the notations given in Figure 4. The set of son-faces of $E$ is supposed to be $\sigma(E)=\left\{E_{1}, E_{2}, E_{3}, E_{4}\right\}$. For a given index $m \in\{1,2,3,4\}$ we define $K_{m}:=K\left(E_{m}\right)$ and the index $m^{\prime}$ as

$$
m^{\prime}:=m-1 \quad \forall m \in\{2,3,4\} \quad \text { and } \quad m^{\prime}:=4 \text { for } m=1 .
$$

Then, the boundary of element $K_{m}$ contains the faces $E_{m}, S_{m}$ and $S_{m^{\prime}}$. Considering the definition (42) of $I_{h}^{E} v$, taking into account the local support of the bubble functions $\left\{\Phi_{S_{m}}\right\}_{1 \leq m \leq 4}$, and using that $\Phi_{E}(x)=0$ for all $x \in \partial K_{m} \backslash\left(E_{m} \cup S_{m} \cup S_{m^{\prime}}\right)$, we obtain by a simple calculation the following equivalent form to the condition (44) for $\widetilde{E}=E_{m}$

$$
\begin{aligned}
& c_{E, S_{m}}(v)\left\langle\phi_{S_{m}} \cdot n^{K_{m}}, 1\right\rangle_{\partial K_{m}}+c_{E, S_{m^{\prime}}}(v)\left\langle\phi_{S_{m^{\prime}}} \cdot n^{K_{m}}, 1\right\rangle_{\partial K_{m}} \\
& =\left\langle v \cdot n_{E_{m}}^{K_{m}}, 1\right\rangle_{E_{m}}-c_{E}(v)\left\langle\Phi_{E} \cdot n^{K_{m}}, 1\right\rangle_{E_{m} \cup S_{m} \cup S_{m^{\prime}}},
\end{aligned}
$$


for all $m \in\{1,2,3,4\}$. In matrix form these equations lead to

$$
\left(\begin{array}{cccc}
f_{1} & 0 & 0 & -f_{4} \\
-f_{1} & f_{2} & 0 & 0 \\
0 & -f_{2} & f_{3} & 0 \\
0 & 0 & -f_{3} & f_{4}
\end{array}\right)\left(\begin{array}{l}
c_{E, S_{1}}(v) \\
c_{E, S_{2}}(v) \\
c_{E, S_{3}}(v) \\
c_{E, S_{4}}(v)
\end{array}\right)=\left(\begin{array}{l}
b_{1} \\
b_{2} \\
b_{3} \\
b_{4}
\end{array}\right)
$$

where $f_{m}$ denotes the normal flux

$$
f_{m}:=\left\langle\Phi_{S_{m}} \cdot n^{K_{m}}, 1\right\rangle_{S_{m}} \quad \forall m \in\{1,2,3,4\},
$$

and

$$
b_{m}:=\left\langle v \cdot n_{E_{m}}^{K_{m}}, 1\right\rangle_{E_{m}}-c_{E}(v)\left\langle\Phi_{E} \cdot n^{K_{m}}, 1\right\rangle_{E_{m} \cup S_{m} \cup S_{m^{\prime}}},
$$

for all $m \in\{1,2,3,4\}$. By the property (28) of the face bubble functions $\left\{\Phi_{S_{m}}\right\}_{1 \leq m \leq 4}$ in the case $d=3$ and the estimate (12) between $h_{K_{m}}$ and $h_{K}$ we have

$$
\left|f_{m}\right| \geq C h_{K_{m}}^{2} \geq C h_{K}^{2} \quad \forall m \in\{1,2,3,4\} .
$$

One can easily check that

$$
\begin{aligned}
c_{E, S_{m}}(v) & =\frac{1}{f_{m}}\left(\sum_{i=1}^{m} b_{i}\right), \quad \forall m \in\{1,2,3\}, \\
c_{E, S_{4}}(v) & =0
\end{aligned}
$$

is an explicit solution of the linear system (46). For each $m \in\{1,2,3,4\}$, we can estimate $b_{m}$ using (26) and the estimate (12) between $h_{K_{m}}$ and $h_{K}$

$$
\begin{aligned}
\left|b_{m}\right| & \leq\left|E_{m}\right|^{1 / 2}\|v\|_{L^{2}\left(E_{m}\right)}+\left|c_{E}(v)\right| \max _{x \in \bar{\Omega}}\left\|\Phi_{E}(x)\right\|\left\{\left|E_{m}\right|+\left|S_{m}\right|+\left|S_{m^{\prime}}\right|\right\} \\
& \leq C h_{K_{m}}\|v\|_{L^{2}(E)}+C h_{K_{m}}^{2}\left|c_{E}(v)\right| \\
& \leq C h_{K}\|v\|_{L^{2}(E)}+C h_{K}^{2}\left|c_{E}(v)\right| .
\end{aligned}
$$

Together with (47) this proves the estimate (45).

\subsubsection{Divergence preserving property of $I_{h}$}

Lemma 7. Let $\mathcal{T}$ be a shape-regular and 1-irregular mesh. The operator $I_{h}$ that has been defined in (23) by means of the local operators $I_{h}^{E}$, developed in Section 2.1.1 for the two dimensional case and in Section 2.1.2 for the three dimensional case, is divergence preserving with respect to the test space $M_{h}^{0}$, i.e. for $v \in\left(H_{0}^{1}(\Omega)\right)^{d}$ it holds

$$
\left(\operatorname{div} I_{h} v, q\right)=(\operatorname{div} v, q) \quad \forall q \in M_{h}^{0} .
$$

Proof. We denote by $q_{K}$ the value of $q \in M_{h}^{0}$ on the element $K \in \mathcal{T}$. Then, using partial integration and the properties (36), (38), (39) for the two dimensional case (resp. (36), (43), (44) for the three dimensional case) of 
the operator $I_{h}^{E}$ we have

$$
\begin{aligned}
& (\operatorname{div} v, q)=\sum_{K \in \mathcal{T}} \sum_{E \in \mathcal{E}(K)} q_{K}\left\langle v \cdot n_{E}^{K}, 1\right\rangle_{E}=\sum_{E \in \mathcal{E}_{0}} \sum_{K \in \mathcal{T}(E)} q_{K}\left\langle v \cdot n_{E}^{K}, 1\right\rangle_{E} \\
& =\sum_{E \in \mathcal{E}_{r}}\left\{q_{K(E)}\left\langle v \cdot n_{E}^{K(E)}, 1\right\rangle_{E}+q_{K^{\prime}(E)}\left\langle v \cdot n_{E}^{K^{\prime}(E)}, 1\right\rangle_{E}\right\} \\
& +\sum_{E \in \mathcal{E}_{i}}\left\{q_{K(E)}\left\langle v \cdot n_{E}^{K(E)}, 1\right\rangle_{E}+\sum_{\widetilde{E} \in \sigma(E)} q_{K(\widetilde{E})}\left\langle v \cdot n_{\widetilde{E}}^{K(\widetilde{E})}, 1\right\rangle_{\widetilde{E}}\right\} \\
& =\sum_{E \in \mathcal{E}_{r}}\left\{q_{K(E)}\left\langle I_{h}^{E} v \cdot n^{K(E)}, 1\right\rangle_{\partial K(E)}+q_{K^{\prime}(E)}\left\langle I_{h}^{E} v \cdot n^{K^{\prime}(E)}, 1\right\rangle_{\partial K^{\prime}(E)}\right\} \\
& +\sum_{E \in \mathcal{E}_{i}}\left\{q_{K(E)}\left\langle I_{h}^{E} v \cdot n^{K(E)}, 1\right\rangle_{\partial K(E)}+\sum_{\widetilde{E} \in \sigma(E)} q_{K(\widetilde{E})}\left\langle I_{h}^{E} v \cdot n^{K(\widetilde{E})}, 1\right\rangle_{\partial K(\widetilde{E})}\right\} \\
& =\sum_{E \in \mathcal{E}_{r}}\left\{\left(\operatorname{div} I_{h}^{E} v, q\right)_{K(E) \cup K^{\prime}(E)}\right\}+\sum_{E \in \mathcal{E}_{i}}\left\{\left(\operatorname{div} I_{h}^{E} v, q\right)_{K(E)}+\sum_{\widetilde{E} \in \sigma(E)}\left(\operatorname{div} I_{h}^{E} v, q\right)_{K(\widetilde{E})}\right\} .
\end{aligned}
$$

Using the fact that the support of $I_{h}^{E} v$ is $\overline{K(E)} \cup \overline{K^{\prime}(E)}$ in the case $E \in \mathcal{E}_{r}$ and $\overline{K(E)} \cup\left(\bigcup_{\widetilde{E} \in \sigma(E)} \overline{K(\widetilde{E})}\right)$ in the case $E \in \mathcal{E}_{i}$ and applying the definition (23) of the operator $I_{h}$ we get

$$
(\operatorname{div} v, q)=\sum_{E \in \mathcal{E}_{r}}\left(\operatorname{div} I_{h}^{E} v, q\right)+\sum_{E \in \mathcal{E}_{i}}\left(\operatorname{div} I_{h}^{E} v, q\right)=\left(\operatorname{div} I_{h} v, q\right)
$$

\subsubsection{Stability properties of $I_{h}$}

In order to establish the needed stability properties of the interpolation operator $I_{h}$ we need first its representation on a given element $K \in \mathcal{T}$. Due to its construction, the interpolation $I_{h}^{E} v$ of $v \in\left(H_{0}^{1}(\Omega)\right)^{d}$ has a local support, i.e.

$$
\operatorname{supp}\left(I_{h}^{E} v\right) \subseteq\left\{\begin{array}{l}
\overline{K(E)} \cup \overline{K^{\prime}(E)}, \text { if } E \in \mathcal{E}_{r} \\
\overline{K(E)} \cup \underset{\widetilde{E} \in \sigma(E)}{\bigcup} \overline{K(\widetilde{E})}, \quad \text { if } E \in \mathcal{E}_{i} .
\end{array}\right.
$$

This leads to the following local representation of $I_{h} v$

$$
\left.I_{h} v\right|_{K}=\left.\sum_{E \in \mathcal{E}_{r}(K) \cup \mathcal{E}_{i}(K)} I_{h}^{E} v\right|_{K}+\left.\sum_{\widetilde{E} \in \mathcal{E}_{\sigma}(K)} I_{h}^{\mathcal{F}(\widetilde{E})} v\right|_{K}
$$

Lemma 8. Let $\mathcal{T}$ be a shape-regular and1-irregular mesh. Let $K \in \mathcal{T}$ and $I_{h}$ the interpolation operator defined in (23). Then, for $v \in\left(H_{0}^{1}(\Omega)\right)^{d}$ it holds

$$
\left|I_{h} v\right|_{1, K}^{2} \leq C \sum_{\widetilde{K} \in \Lambda(K)}\left\{h_{\widetilde{K}}^{-2}\|v\|_{0, \widetilde{K}}^{2}+|v|_{1, \widetilde{K}}^{2}\right\}
$$

with $\Lambda(K)$ from (10) and

$$
\left|I_{h} v\right|_{1, \Omega}^{2} \leq C \sum_{K \in \mathcal{T}}\left\{h_{K}^{-2}\|v\|_{0, K}^{2}+|v|_{1, K}^{2}\right\} .
$$




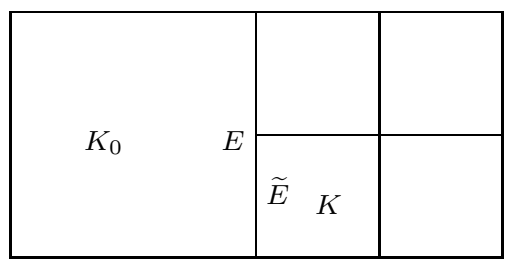

Figure 5. Two dimensional configuration for the case of $\widetilde{E} \in \mathcal{E}_{\sigma}(K), E:=\mathcal{F}(\widetilde{E})$ and $K_{0}:=K(E)$.

Proof. First we prove the estimate (51). Let $E \in \mathcal{E}_{r}(K) \cup \mathcal{E}_{i}(K)$ for a given $K \in \mathcal{T}$. Due to (34), (37) and (42) we have

$$
\left.I_{h}^{E} v\right|_{K}=\left.c_{E}(v) \phi_{E}\right|_{K}
$$

where we have used the fact that for $E \in \mathcal{E}_{i}(K)$ we have $\left.\phi_{S_{m}}\right|_{K}=0$ for all $m=1, \ldots, 4^{d-2}$. Due to (35) and (28), we get for $c_{E}(v)$

$$
\begin{aligned}
\left|c_{E}(v)\right| & =\frac{\left\langle v \cdot n_{E}, 1\right\rangle_{E}}{\left\langle\Phi_{E} \cdot n_{E}, 1\right\rangle_{E}} \leq \frac{|E|^{1 / 2}\|v\|_{0, E}}{C_{3} h_{K}^{d-1}} \\
& \leq \frac{C_{5}^{1 / 2} C_{4}}{C_{3}} h_{K}^{-d / 2+1}\left\{h_{K}^{-1}\|v\|_{0, K}+|v|_{1, K}\right\}
\end{aligned}
$$

where the last estimate follows from (11) and (29). Together with (27) we obtain

$$
\left|I_{h}^{E} v\right|_{1, K} \leq C_{10}\left\{h_{K}^{-1}\|v\|_{0, K}+|v|_{1, K}\right\} \quad \forall E \in \mathcal{E}_{r}(K) \cup \mathcal{E}_{i}(K),
$$

with $C_{10}:=C_{5}^{1 / 2} C_{4} C_{2} / C_{3}$.

Now, we consider the configuration corresponding to the Figure 5 i.e. $\widetilde{E} \in \mathcal{E}_{\sigma}(K), E:=\mathcal{F}(\widetilde{E})$ and $K_{0}:=$ $K(E)$. Based on the representation (50), we obtain

$$
\left.I_{h}^{E} v\right|_{K}=\left.c_{E}(v) \Phi_{E}\right|_{K}+\left.\sum_{m=1}^{4^{d-2}} c_{E, S_{m}}(v) \Phi_{S_{m}}\right|_{K}
$$

Since $E \in \mathcal{E}\left(K_{0}\right)$, we get similarly to $(53)$

$$
\left|c_{E}(v)\right| \leq \frac{C_{5}^{1 / 2}}{C_{3}} h_{K_{0}}^{-(d-1) / 2}\|v\|_{0, E} .
$$

Using (27), (12) and (29) we get

$$
\begin{aligned}
\left|c_{E}(v) \Phi_{E}\right|_{1, K} & \leq \frac{C_{5}^{1 / 2} C_{2} C_{7}^{\frac{d}{2}-1}}{C_{3}} h_{K_{0}}^{-1 / 2}\|v\|_{0, E} \\
& \leq \frac{C_{5}^{1 / 2} C_{2} C_{7}^{\frac{d}{2}-1} C_{4}}{C_{3}}\left\{h_{K_{0}}^{-1}\|v\|_{0, K_{0}}+|v|_{1, K_{0}}\right\} .
\end{aligned}
$$

Based on the estimates (41) and (45) we deduce similarly the following estimates

$$
\begin{aligned}
\left|c_{E, S_{m}}(v)\right| \leq & C_{8} h_{K_{0}}^{-(d-1) / 2}\|v\|_{L^{2}(E)}+C_{9}\left|c_{E}(v)\right|, \\
& \leq C h_{K_{0}}^{1-\frac{d}{2}}\left\{h_{K_{0}}^{-1}\|v\|_{0, K_{0}}+|v|_{1, K_{0}}\right\} .
\end{aligned}
$$


Together with (27) we then obtain

$$
\left|c_{E, S_{m}}(v) \Phi_{S_{m}}\right|_{1, K} \leq C\left\{h_{K_{0}}^{-1}\|v\|_{0, K_{0}}+|v|_{1, K_{0}}\right\},
$$

for all $m \in\left\{1, \ldots, 4^{d-2}\right\}$. Finally from (56), (59) and (61) we get

$$
\left|I_{h}^{E} v\right|_{1, K} \leq C\left\{h_{K_{0}}^{-1}\|v\|_{0, K_{0}}+|v|_{1, K_{0}}\right\} \quad \text { where } K_{0}=K(E) .
$$

Using the representation formula (50) of $\left.I_{h} v\right|_{K}$ we obtain

$$
\begin{aligned}
\left|I_{h} v\right|_{1, K}^{2} & \leq C\left\{h_{K}^{-2}\|v\|_{0, K}^{2}+|v|_{1, K}^{2}\right\}+C \sum_{\widetilde{E} \in \mathcal{E}_{\sigma}(K)}\left\{h_{K(\mathcal{F}(\widetilde{E}))}^{-2}\|v\|_{0, K(\mathcal{F}(\widetilde{E}))}^{2}+|v|_{1, K(\mathcal{F}(\widetilde{E}))}^{2}\right\} \\
& \leq C \sum_{\widetilde{K} \in \Lambda(K)}\left\{h_{\widetilde{K}}^{-2}\|v\|_{0, \widetilde{K}}^{2}+|v|_{1, \widetilde{K}}^{2}\right\}
\end{aligned}
$$

which concludes the proof of (51). The estimate (52) is a direct consequence of (51).

\section{2. $H^{1}$-stable interpolation operator $R_{h}$}

The definition of the interpolation operator $\Pi_{h}$ in (22) also requires the construction of an interpolation operator $R_{h}:\left(H_{0}^{1}(\Omega)\right)^{d} \rightarrow X_{h}^{1}$ which aims at approximating possibly non-smooth functions in $H^{1}$ by means of continuous piecewise polynomials of low order. Such an operator for $H^{1}$ functions has been proposed by Scott and Zhang [22] and its construction is based on averaging over $(d-1)$-dimensional faces of the $d$-dimensional elements. However, the restriction of this approach is that it assumes affine equivalent elements and a regular mesh which excludes hanging nodes. In [17] an extension of the Scott-Zhang operator for the case of a shape regular and 1-irregular mesh is proposed and the following properties have been proven.

Lemma 9. Let $\mathcal{T}$ be a shape-regular and 1-irregular mesh. There exists an interpolation operator $R_{h}$ such that for $K \in \mathcal{T}$ it holds

where

$$
\begin{gathered}
\left|R_{h} v\right|_{1, K} \leq C|v|_{1, \omega(K)} \quad \forall v \in\left(H^{1}(\Omega)\right)^{d} \\
\left\|v-R_{h} v\right\|_{0, K} \leq C h_{K}|v|_{1, \omega(K)} \quad \forall v \in\left(H^{1}(\Omega)\right)^{d}
\end{gathered}
$$

$$
\delta(K):=\bigcup_{\widetilde{K} \in \Lambda(K)} \overline{\widetilde{K}}, \quad \omega(K):=\bigcup_{\widetilde{K} \in \delta(K)} \delta(\widetilde{K}) .
$$

Proof. See [17], Theorem 8.

\subsection{Properties of $\Pi_{h}$}

Now, for the operator $\Pi_{h}:\left(H_{0}^{1}(\Omega)\right)^{d} \rightarrow X_{h}^{2}$ defined in (22) by means of the operators $I_{h}:\left(H_{0}^{1}(\Omega)\right)^{d} \rightarrow X_{h}^{2}$ and $R_{h}:\left(H_{0}^{1}(\Omega)\right)^{d} \rightarrow X_{h}^{1}$ with $X_{h}^{1} \subset X_{h}^{2}$, we are able to prove the needed properties (20) and (21).

Lemma 10. Let $\mathcal{T}$ be a shape-regular and 1-irregular mesh. Then, the operator $\Pi_{h}$ defined in $(22)$ is $H^{1}$-stable uniformly with respect to $h$ in the sense of (20) and divergence preserving in the sense of (21).

Proof. From the definition (22) of $\Pi_{h}$, the stability of $R_{h}$ in (63) and of $I_{h}$ in (52) we obtain

$$
\begin{aligned}
\left|\Pi_{h} v\right|_{1, \Omega}^{2} & \leq 2\left|R_{h} v\right|_{1, \Omega}^{2}+2\left|I_{h}\left(v-R_{h} v\right)\right|_{1, \Omega}^{2}, \\
& \leq C\left\{|v|_{1, \Omega}^{2}+\sum_{K \in \mathcal{T}}\left\{h_{K}^{-2}\left\|v-R_{h} v\right\|_{0, K}^{2}+\left|v-R_{h} v\right|_{1, K}^{2}\right\}\right\}, \\
& \leq C|v|_{1, \Omega}^{2},
\end{aligned}
$$


where the last estimate relies on the interpolation property (64) of $R_{h}$. This shows that $\Pi_{h}$ is $H^{1}$-stable in the sense of (20). The property (21) that $\Pi_{h}$ is divergence preserving is an easy consequence of the fact that the operator $I_{h}$ is divergence preserving.

\section{PROVING The INF-SUP CONDITION}

Our approach to prove the inf-sup condition is based on the macro-element technique proposed by Boland and Nicolaides [6] (see also [13], Sect. II.1.4). In its general setup, this technique relies on a partitioning of the domain $\Omega$ by means of $R$ nonoverlapping open subdomains $\Omega_{r}, r=1, \ldots, R$, with Lipschitz continuous boundary. In our case, these subdomains $\Omega_{r}$ are built by the mesh elements $K \in \mathcal{T}$. Based on the finite element spaces $X^{N}=\left(S^{N}\right)^{d}$ and $M^{N}$ defined in (14) and (15), respectively, we define the following local spaces

$$
\begin{aligned}
& X^{N}(K):=\left\{v \in\left(H_{0}^{1}(K)\right)^{d}: v \circ F_{K} \in\left(\mathbb{Q}_{r_{K}}(\widehat{K})\right)^{d}\right\} \\
& M^{N}(K):=\left\{q \in L_{0}^{2}(K): q \circ F_{K} \in \mathbb{P}_{r_{K}-1}(\widehat{K})\right\}
\end{aligned}
$$

We say that the finite element spaces $X^{N}$ and $M^{N}$ fulfill the local inf-sup condition uniformly with respect to $K \in \mathcal{T}$ if there exist constants $\lambda\left(r_{K}\right)>0$, such that for all $K \in \mathcal{T}$ it holds

$$
\inf _{q^{N} \in M^{N}(K) \backslash\{0\}} \sup _{v^{N} \in X^{N}(K)} \frac{\left(\nabla \cdot v^{N}, q^{N}\right)_{K}}{\left|v^{N}\right|_{1, K}\left\|q^{N}\right\|_{0, K}} \geq \lambda\left(r_{K}\right),
$$

where $\lambda\left(r_{K}\right)$ is independent of the mesh cell $K$ and the mesh parameter $h$ but may depend on the polynomial degree $r_{K}$ of the local spaces $X^{N}(K)$ and $M^{N}(K)$.

Now, in order to prove the global inf-sup condition for our finite element spaces $X^{N}=\left(S^{N}\right)^{d}$ and $M^{N}$, defined in (14) and (15), respectively, we only have to verify the local inf-sup condition (67) and the global inf-sup condition for a suitable pair of low order subspaces $\left(\bar{X}^{N}, M_{h}^{0}\right)$. This is justified by the following lemma.

Lemma 11. Let the local inf-sup condition (67) be fulfilled uniformly with respect to $K \in \mathcal{T}$ with positive constants $\lambda\left(r_{K}\right)$ independent of $K$ and the mesh parameter $h$. Furthermore, we assume that there exists a subspace $\bar{X}^{N} \subset X^{N}$ such that the pair of finite element spaces $\left(\bar{X}^{N}, M_{h}^{0}\right)$ fulfills the global inf-sup condition

$$
\inf _{q_{h} \in M_{h}^{0} \backslash\{0\}} \sup _{v_{h} \in \bar{X}^{N}} \frac{\left(\nabla \cdot v_{h}, q_{h}\right)_{\Omega}}{\left|v_{h}\right|_{1, \Omega}\left\|q_{h}\right\|_{0, \Omega}} \geq \bar{\beta}>0,
$$

with a constant $\bar{\beta}>0$ independent of $h$. Then, the pair $\left(X^{N}, M^{N}\right)$ satisfies the global inf-sup condition (2) with a constant $\beta>0$ which depends on $\bar{\beta}$ and on

$$
\lambda^{*}:=\min _{r=2, \ldots, r^{*}} \lambda(r), \quad \text { where } r^{*}:=\max _{K \in \mathcal{T}} r_{K}
$$

but which is independent of $h$.

Proof. Due to the definition of $\lambda^{*}$ in (69), the local inf-sup condition (67) is satisfied with the fixed positive constant $\lambda^{*}$ for each element $K \in \mathcal{T}$. Therefore, the statement of Lemma 11 directly follows from Theorem II.1.12 in $[13]$.

A nice feature in the analysis of our pair of higher order finite element spaces $\left(X^{N}, M^{N}\right)$ on a 1-irregular mesh with hanging nodes is that the local spaces $X^{N}\left(K_{0}\right)$ and $M^{N}\left(K_{0}\right)$ for a fixed element $K_{0} \in \mathcal{T}$ with the polynomial degree $r_{0}=r_{K_{0}}$ are completely the same as in the case of a higher order pair $\left(X_{h}, M_{h}\right)$ based on the uniform polynomial degree $r_{0}$ and a mesh $\mathcal{T}_{h}$ without hanging nodes that contains $K_{0}$. Therefore, the proof of the local inf-sup condition (67) for our local spaces defined in (65) and (66) is the same as in [19]. 
Lemma 12. Let $\mathcal{T}$ be a shape-regular mesh. Then, for each element $K \in \mathcal{T}$, the pair $\left(X^{N}(K), M^{N}(K)\right)$ of the mapped local finite element spaces defined in (65) and (66), satisfies the local inf-sup condition (67) with a constant $\lambda\left(r_{K}\right)>0$ which is independent of $K$ and the mesh parameter $h$.

Proof. See the proof of Theorem 8 in [19].

The global inf-sup condition (68) of the pair of spaces $\left(\bar{X}^{N}, M_{h}^{0}\right)$ can be proved for the second order subspace $\bar{X}^{N}=X_{h}^{2} \subset X^{N}$.

Lemma 13. Let $\mathcal{T}$ be a locally refined mesh, which is assumed to be 1-irregular and shape-regular. Then, the pair of finite element spaces $\left(\bar{X}^{N}, M_{h}^{0}\right)$ with $\bar{X}^{N}:=X_{h}^{2}$ satisfies the global inf-sup condition (68) with a constant $\bar{\beta}>0$ independent of $h$.

Proof. In Section 2.3, we have constructed an interpolation operator $\Pi_{h}:\left(H_{0}^{1}(\Omega)\right)^{d} \rightarrow X_{h}^{2}$ which is $H^{1}$-stable uniformly with respect to $h$ (see (20)) and divergence preserving (see (21)). From these properties, due to Fortin [12] (see also [13], Lem. II.1.1), we deduce that the inf-sup condition (68) holds.

Now, we can state our main result.

Theorem 14. Let $\mathcal{T}$ be locally refined mesh which is shape-regular and 1-irregular in the sense of Definitions 1 and 3 , respectively. For the pair of finite element spaces $\left(X^{N}, M^{N}\right)$, defined in (14) and (15) with $X^{N}=\left(S^{N}\right)^{d}$, it holds the global inf-sup condition (2) with an $h$-independent constant $\beta>0$ which depends on the constants $\bar{\beta}$ from Lemma 13 and $\lambda^{*}$ defined in (69) with the constants $\lambda(r)$ from Lemma 12.

Proof. The proof of Theorem 14 is a direct consequence of Lemmas 11, 12 and 13.

Remark 15. Theorem 14 shows that, even for a locally refined, 1-irregular and shape regular mesh $\mathcal{T}$, the infsup constant $\beta$ in (2) is independent of the local and global mesh parameters $h_{K}$ and $h$, respectively. However, the dependency of $\beta$ on the polynomial degree vector $\mathbf{r}:=\left\{r_{K}: K \in \mathcal{T}\right\}$ is not investigated. For the situation of just one cell $\mathcal{T}=\left\{K_{0}\right\}$ with $K_{0}=(-1,1)^{d}$, it has been shown in [4] that the inf-sup constant $\beta$ is independent of the polynomial degree $r=r_{K_{0}}$ of the spaces $X^{N}\left(K_{0}\right)$ and $M^{N}\left(K_{0}\right)$ defined in $(65)$ and (66). The proof that the local inf-sup-constants $\lambda\left(r_{K}\right)$ in (67) are independent of the polynomial degree $r_{K}$ on grids described in Section 1.2 with a sufficiently small mesh size $h \leq h_{0}$ will be the object of a forthcoming paper.

Remark 16. For the pair of finite element spaces $X^{N}$ and $\widetilde{M}^{N}$ with the so-called unmapped pressure space defined in [13], Section II.3.2, we can derive an analogous result as in Theorem 14. To this end, we need the local inf-sup condition (67) with the local unmapped pressure space

$$
\widetilde{M}^{N}(K):=\left\{q \in L_{0}^{2}(K): q \in \mathbb{P}_{r_{K}-1}(K)\right\}
$$

instead of the local mapped space $M^{N}(K)$ defined in (66). Again the inf-sup constants $\lambda\left(r_{K}\right)$ have to be independent of $K$ and the mesh parameter $h$. This local inf-sup condition has been proven in [13] Theorem II.3.2, for the 2D-case and the proof can be generalized in a straightforward way to the 3D-case. The global inf-sup condition (68) for the low order subspaces $\left(\bar{X}^{N}, M_{h}^{0}\right) \subset\left(X^{N}, \widetilde{M}^{N}\right)$ in case of the unmapped pressure space is exactly the same as in the case of the mapped pressure space $M^{N}$. Therefore, this condition follows from Lemma 13. Thus, applying Lemma 11, Theorem II.3.2 in [13] and its generalization to the 3D-case, we obtain the analogous result as in Theorem 14 for the pair of finite element spaces $\left(X^{N}, \widetilde{M}^{N}\right)$.

\section{REFERENCES}

[1] M. Ainsworth and P. Coggins, A uniformly stable family of mixed $h p$-finite elements with continuous pressures for incompressible flow. IMA J. Numer. Anal. 22 (2002) 307-327.

[2] I. Babuška and M. Suri, The $p$ and $h-p$ versions of the finite element method, basic principles and properties. SIAM Rev. 36 (1994) 578-632 
[3] C. Bernardi and Y. Maday. Approximations spectrales de problèmes aux limites elliptiques. (Spectral approximation for elliptic boundary value problems). Mathématiques \& Applications, Paris, Springer-Verlag 10 (1992).

[4] C. Bernardi and Y. Maday, Uniform inf-sup conditions for the spectral discretization of the Stokes problem. Math. Models Methods Appl. Sci. 9 (1999) 395-414.

[5] D. Boffi and L. Gastaldi, On the quadrilateral $\mathrm{Q}_{2}-\mathrm{P}_{1}$ element for the Stokes problem. Int. J. Numer. Methods Fluids 39 (2002) 1001-1011.

[6] J.M. Boland and R.A. Nicolaides, Stability of finite elements under divergence constraints. SIAM J. Numer. Anal. 20 (1983) $722-731$.

[7] S. Bönisch, V. Heuveline and P. Wittwer, Adaptive boundary conditions for exterior flow problems. J. Math. Fluid Mech. 7 (2005) 85-107.

[8] F. Brezzi and R.S. Falk, Stability of higher-order Hood-Taylor methods. SIAM J. Numer. Anal. 28 (1991) 581-590.

[9] F. Brezzi and M. Fortin, Mixed and Hybrid Finite Element Methods. Springer Series in Computational Mathematics, SpringerVerlag 15 (1991).

[10] L. Chilton and M. Suri, On the construction of stable curvilinear $p$ version elements for mixed formulations of elasticity and Stokes flow. Numer. Math. 86 (2000) 29-48.

[11] P.G. Ciarlet, The finite element method for elliptic problems. Studies in Mathematics and its Applications 4, Amsterdam New York - Oxford: North-Holland Publishing Company (1978).

[12] M. Fortin, An analysis of the convergence of mixed finite element methods. RAIRO Anal. Numer. 11 (1977) 341-354.

[13] V. Girault and P.-A. Raviart, Finite Element Methods for Navier-Stokes Equations. Springer-Verlag, Berlin-Heidelberg-New York (1986).

[14] V. Heuveline and M. Hinze, Adjoint-based adaptive time-stepping for partial differential equations using proper orthogonal decomposition. Technical report, University Heidelberg, SFB 359 (2004).

[15] V. Heuveline and R. Rannacher, A posteriori error control for finite element approximations of elliptic eigenvalue problems. Adv. Comput. Math. 15 (2001) 107-138.

[16] V. Heuveline and R. Rannacher, Duality-based adaptivity in the $h p$-finite element method. J. Numer. Math. 11 (2003) 95-113.

[17] V. Heuveline and F. Schieweck, An interpolation operator for $H^{1}$ functions on general quadrilateral and hexahedral meshes with hanging nodes. Technical report, University Heidelberg, SFB 359 (2004).

[18] G. Matthies, Mapped finite elements on hexahedra. Necessary and sufficient conditions for optimal interpolation errors. Numer. Algorithms 27 (2001) 317-327.

[19] G. Matthies and L. Tobiska, The inf-sup condition for the mapped $Q_{k}-P_{k-1}^{\text {disc }}$ element in arbitrary space dimensions. Computing 69 (2002) 119-139.

[20] S. Schötzau, C. Schwab and R. Stenberg, Mixed $h p$-fem on anisotropic meshes. II: Hanging nodes and tensor products of boundary layer meshes. Numer. Math. 83 (1999) 667-697.

[21] Ch. Schwab, $p$ - and $h p$-finite element methods. Theory and applications in solid and fluid mechanics. Numerical Mathematics and Scientific Computation, Oxford: Clarendon Press (1998).

[22] L.R. Scott and S. Zhang, Finite element interpolation of nonsmooth functions satisfying boundary conditions. Math. Comp. 54 (1990) 483-493.

[23] R. Stenberg, Error analysis of some finite element methods for the Stokes problem. Math. Comp. 54 (1990) 495-508.

[24] R. Stenberg and M. Suri, Mixed $h p$ finite element methods for problems in elasticity and Stokes flow. Numer. Math. 72 (1996) 367-389.

[25] L. Stupelis, Navier-Stokes equations in irregular domains. Mathematics and its Applications 326, Dordrecht: Kluwer Academic Publishers (1995). 